\title{
INTERPOLATION BETWEEN CONSECUTIVE CONJUGATE POINTS OF AN $n$ TH ORDER LINEAR DIFFERENTIAL EQUATION
}

\author{
BY
}

\section{G. B. GUSTAFSON}

\begin{abstract}
The interpolation problem $x^{(n)}+P_{n-1} x^{(n-1)}+\cdots+P_{0} x=0$, $x^{(i)}\left(t_{j}\right)=0, i=0, \cdots, k_{j}-1, j=0, \cdots, m$, is studied on the conjugate interval $\left[a, \eta_{1}(a)\right]$. The main result is that there exists an essentially unique nontrivial solution of the problem almost everywhere, provided $k_{1}+\cdots+k_{m} \geq n$, and certain other inequalities are satisfied, with $a=t_{0}<t_{1}<\ldots<t_{m}=\eta_{1}(a)$.

In particular, this paper corrects the results of Azbelev and Caljuk (Mat. Sb. 51 (93) (1960), 475-486; English transl., Amer. Math. Soc. Transl. (2) 42 (1964), 233-245) on third order equations, and shows that their results are correct almost everywhere.
\end{abstract}

1. Introduction. An interpolation problem for the $n$th order linear differential equation

$$
L x \equiv x^{(n)}+\sum_{k=0}^{n-1} p_{k}(t) x^{(k)}=0
$$

is the problem of finding a solution $x(t)$ of $L x=0$ satisfying the multiple point relations

$$
x^{(i)}\left(t_{j}\right)=c_{i j}, \quad i=0, \ldots, k_{j}-1, j=0, \cdots, m .
$$

The coefficients in (1.1) are assumed continuous on $(-\infty, \infty)$, or if $L^{*}$ is to be defined, $p_{k}(t)$ is assumed of class $C^{k}, 0 \leq k \leq n-1$. The points $t_{0}, \cdots, t_{m}$ are assumed to be in strictly increasing order, and the numbers $k_{j}$ and $c_{i j}$ are arbitrary, subject to $k_{j} \leq n-1$. The investigation here is directed toward the zero problem, i.e., all $c_{i j}=0$.

To simplify notation, write $k=\left(k_{0}, \cdots, k_{m}\right), T=\left\{t_{0}, \cdots, t_{m}\right\}$; the statement " $x(t)$ has a zero of order $k$ at $T$ " will replace " $x(t)$ is a nontrivial solution of (1.1), (1.2) with all $c_{i j}=0$ ''. The symbol $|k|$ is defined by $|k|=k_{0}+\cdots+k_{m}$; hence $x(t)$ has at least $|k|$ zeros, counting multiplicities, on $\left[t_{0}, t_{m}\right]$. The solution $x(t)$ is said to have a zero of order exactly $k$ at $T$, or exactly $|k|$ zeros on $\left[t_{0}, t_{m}\right]$ if $x(t)$ has the zeros specified, but no others on $\left[t_{0}, t_{m}\right]$, counting multiplicities.

Received by the editors February 8, 1972.

AMS (MOS) subject classifications (1970). Primary 34B05, 34B10; Secondary 34C10. Key words and phrases. Disconjugacy, interpolation, first conjugate point. 
The equation $L x=0$ is disconjugate on a connected set $E$ if for all solutions $x(t) \equiv 0$ with a zero of order $k$ at $T \subseteq E$ it follows that $|k|<n$; otherwise, $L x=0$ is said to be conjugate on $E$.

In the case of conjugacy on $[a, \infty)$, one can define with Leighton and Nehari [5] and Sherman [9] a first conjugate point $\eta_{1}(a)$ to the point $a: \eta_{1}(a)$ is the minimum of all points $b>a$ such that $L x=0$ is conjugate on $[a, b]$. In [10], Sherman proves that $\eta_{1}(a)$ is a continuous, strictly increasing function of $a$, whose domain is of the form $(-\infty, d)$, where $d \leq+\infty$. Furthermore, it is shown in [9] that there exists a solution $x(t)$ with a zero of order $(p, n-p)$ at $\left\{a, \eta_{1}(a)\right\}$ with $x(t)$ $>0$ in $\left(a, \eta_{1}(a)\right)$. The largest integer $p$ is denoted $p(a)$, and $q(a)$ is the exact order of the zero at $\eta_{1}(a)$ for the corresponding solution $x(t)$.

The main result can now be simply stated: for almost all $a$ in the domain of $\eta_{1}(t)$, there is an essentially unique solution with a zero of order exactly $k$ at $T$, where $k$ and $T$ satisfy the relations

$$
\begin{aligned}
& a=t_{0}<t,<\cdots<t_{m}=\eta_{1}(a), \\
& p(a)=|k|-k_{m}, \quad q(a)=|k|-k_{0}, \\
& p(a)+q(a)-n \geq|k|-k_{0}-k_{m} .
\end{aligned}
$$

In the applications, equations $L x=0$ are exhibited such that $p(a)=q(a)=$ $n-1$ for every point, and the main theorem just stated allows the following conclusion: for all points $a$, there is a solution with precisely $n$ simple zeros on $\left[a, \eta_{1}(a)\right]$.

The proof of the main result depends heavily on differentiability properties of $\eta_{1}(t)$, and in article 2 the necessary preliminaries are presented. Fortunately, sophisticated techniques are not needed and the proofs presented here are tedious, but elementary.

The results contained herein correct the false statements of Azbelev and Caljuk [1], and show that they were correct almost everywhere. The author is indebted to the late J. H. Barrett, and T. L. Sherman, for their conversations on this subject.

If $T_{n}=\left\{t_{0 n}, t_{1 n}, \cdots, t_{m n}\right\}, T=\left\{t_{0}, \cdots, t_{m}\right\}$ and $\lim _{n \rightarrow \infty} t_{j n}=t_{j}, 0<j-m$, then one writes $T_{n} \rightarrow T$ as $n \rightarrow \infty$.

Convergence Lemma. If $L x=0$ has a solution with a zero of order $k$ at $T_{n}$, and $T_{n} \rightarrow T$ as $n \rightarrow \infty$, then $L x=0$ bas a solution with a zero of order $k$ at $T$.

With the aid of the contraction mapping principle in [3, p. 260], one can prove the following implicit function theorem. It is different from that found in most texts, because of the weak assertion of uniqueness. 
Implicit Function Theorem. Let $H$ and $K$ be open sets with $(a, b) \in H \times K$, and suppose $F(t, s) \in C^{1}(H \times K), F(a, b)=0$.

If $F_{s}(a, b) \neq 0$, then a neigbborbood $L \subseteq H$ of $t=a$ and $a$ continuous function $g: L \rightarrow K$ can be found such that $g(a)=b$, and $F(t, g(t))=0$ for all $t \in L$. Further, for any $b \in C(L)$ with $b(a)=b$, there is a neighborbood $M, a \in M \subseteq L$, such that $b(M) \subseteq K$, and $b(c)=g(c)$ at points $c \in M$ with $F(c, b(c))=0$.

2. Differentiability of $\eta_{1}$. The principal solutions $u_{0}(\cdot, a), \cdots, u_{n-1}(\cdot, a)$ of $L u=0$ are defined by the initial conditions

$$
\left(D^{j} u_{k}\right)(a)=\delta_{k j}, \quad k, j=0, \cdots, n-1 .
$$

Accordingly, the principal solutions form a basis for the solution space of $L u=0$.

The Wronskian of solutions $x_{1}, \cdots, x_{k}$ of $(1.1)$ is the determinant of the $k \times k$ matrix $\left(D^{i-1} x_{j}\right)$ and is denoted by $W\left(x_{1}, \cdots, x_{k}\right)$.

The following lemmas will be used repeatedly. The proofs are omitted.

2.1. Lemma (T. L. Sherman [9]). Let $u_{j}(t)$ bave a zero of order $k_{j}=\left(p_{j}, q_{j}\right)$ at $\{a, b\}, j=1$, 2. If $m=\max \left\{p_{2}, q_{2}\right\}$ and $u_{1}, u_{2} \in C^{m}[a, b], u_{1} u_{2} \neq 0$ in $(a, b)$, then there is a linear combination of $u_{1}$ and $u_{2}$ which bas a double zero in $(a, b)$.

2.2. Lemma. The problem (1.1), (1.2) has a nontrivial solution if $|k|<n$.

Let $q(a)$ be the exact order of the zero at $\eta_{1}(a)$ for an extremal solution on $\left[a, \eta_{1}(a)\right]$. Given a closed interval $F$ in the domain of $\eta_{1}$, the sets

$$
E_{r s}=\{a \in F: p(a)=r, q(a)=s\},
$$

$1 \leq r, s \leq n-1$, exhaust $F$, and by the Baire Category Theorem the closure of one of these sets must contain a segment $G$. This proves the

2.3. Lemma. Let $L x=0$ bave a conjugate function $\eta_{1}$ defined on a closed interval $F \subseteq(-\infty, \infty)$. Then there exists an open interval $G \subseteq F$ and numbers $r, s$ such that

$$
G \subseteq \operatorname{cl}\{a \in G: p(a)=r, q(a)=s\}
$$

It follows immediately from the convergence lemma that $L x=0$ has a nontrivial solution of order $(r, s)$ at $\left\{a, \eta_{1}(a)\right\}$, for every $a \in G$. Suppose $a \in E_{r s} \cap G$. It follows from the following lemma that $\eta_{1} \in C^{2}(U)$ for some neighborhood $U$ of a. The proof appears in article 6 .

2.4. Lemma. Let a belong to the domain of $\eta_{1}$, and assume there is a neighborbood $V$ of a sucb that $L x=0$ bas a nontrivial solution $x$ with a zero of order $(p(a), n-p(a))$ at $\left\{t, \eta_{1}(t)\right\}$ and $\left(D^{q(a)-1} x\right)\left(\eta_{1}(t)\right)=0$, for each $t \in V$. Then there exists a neighborbood $U$ of a such that $\eta_{1} \in C^{2}(U)$. 
In view of the lemma, if $\{a: p(a)=r\}$ is dense in a segment $V$, then there is a closed nowhere dense subset $E \subseteq V$ such that $\eta_{1} \in C^{2}(V-E)$. Hence the conjugate function $\eta_{1}$ is of class $C^{2}$ on a dense open subset of its domain. Since $\eta_{1}$ is increasing, $\eta_{1}$ is differentiable off a nowhere dense set of zero measure.

2.5. Theorem. The conjugate function $\eta_{1}$ is differentiable off a nowbere dense set of zero measure.

2.6. Corollary. The points $t$ satisfying

$$
\lim _{s \rightarrow t} \inf _{\frac{1}{1}(s)-\eta_{1}(t)}=0
$$

constitute a nowbere dense set of zero measure.

2.7. Example. A differential equation such that $\eta_{1}^{\prime}(0)$ fails to exist.

Let $L y=0$ be the third order linear differential equation with $C^{\infty}$ coefficients on $(-\infty, \infty)$ generated by the fundamental solutions $1, \sin t \sin 2 t, \cos t \sin 2 t$. Surely $\eta_{1}(0)=\pi / 2$, and there exist solutions with zeros of orders $(2,1)$ and $(1,2)$ at $\left\{0, \eta_{1}(0)\right\}$. However, there is no solution with a zero of $\operatorname{order}(2,2)$ at $\{0$, $\left.\eta_{1}(0)\right\}$. Let us show that this leads to $\eta_{1} \in C^{2}(U-\{0\})$ for some neighborhood $U$ of 0 , and the relations $\lim _{t \rightarrow 0-} \eta_{1}^{\prime}(t)=\infty, \lim _{t \rightarrow 0+} \eta_{1}^{\prime}(t)=0$. The nonexistence of $\eta_{1}^{\prime}(0)$ then follows by an elementary argument.

First, let us argue that, for small $b>0$, the intervals $(-b, 0)$ and $(0, b)$ satisfy the following condition: the only solution of $L y=0$ with a zero of order $(2,1)$ at $\left\{a, \eta_{1}(a)\right\}, a \in(-b, 0)$, is $y \equiv 0$, and the only solution of $L z=0$ with a zero of order $(1,2)$ at $\left\{b, \eta_{1}(b)\right\}, b \in(0, b)$, is $z \equiv 0$.

Indeed, suppose not. Then, for example, there would be a sequence of points $t_{1}<t_{2}<\cdots$ converging to 0 such that $u_{2}\left(\eta_{1}\left(t_{n}\right), t_{n}\right)=0$. The function $u_{2}\left(t, t_{n}\right)$ converges uniformly on compact subsets to $u_{2}(t, 0)$, and since $u_{2}(t, 0)$ has a simple zero at $\eta_{1}(0), u_{2}\left(t, t_{n}\right)$ has a simple zero at $\eta_{1}\left(t_{n}\right)$ for all large $n$. Let $F(t)=W\left(u_{2}(t, 0), u_{2}\left(t, \eta_{1}(0)\right), u_{2}\left(t, t_{n}\right)\right)$ for fixed $n, n$ large. Then $F(t)$ is onesigned. However,

$$
\begin{gathered}
F(0)=-u_{2}^{\prime \prime}(0,0) u_{2}^{\prime}\left(0, \eta_{1}(0)\right) u_{2}\left(0, t_{n}\right)<0, \\
F\left(\eta_{1}(0)\right)=-u_{2}^{\prime}\left(\eta_{1}(0), 0\right) u_{2}^{\prime \prime}\left(\eta_{1}(0), \eta_{1}(0)\right) u_{2}\left(\eta_{1}(0), t_{n}\right)>0 .
\end{gathered}
$$

The proof for the right neighborhood of 0 is analogous.

The lemma on differentiability of $\eta_{1}$ shows that $\eta_{1}$ is of class $C^{2}$ on $(-b, b)-\{0\}$.

Since $u_{2}\left(t, \eta_{1}(t)\right)=0$, for all $t \in(-b, b)-\{0\}, b$ small, the implicit function theorem and Peano's theorem (see [4, p. 95]) gives 


$$
\eta_{1}^{\prime}(t)=u_{2}^{\prime}\left(t, \eta_{1}(t)\right) / u_{1}\left(t, \eta_{1}(t)\right)
$$

The solution $u_{1}\left(t, \eta_{1}(0)\right)$ is a linear combination of $u_{2}(t, 0)$ and $u_{2}\left(t, \eta_{1}(0)\right)$, and therefore vanishes at 0 . Accordingly, $\eta_{1}^{\prime}(t) \rightarrow \infty$ as $t \rightarrow 0-$. Similarly,

$$
\eta_{1}^{\prime}(t)=u_{1}\left(\eta_{1}(t), t\right) / u_{2}^{\prime}\left(\eta_{1}(t), t\right)
$$

in a left neighborhood of 0 , and $\eta_{1}^{\prime}(t) \rightarrow 0$ as $t \rightarrow 0+$.

It also follows that $\eta_{1}^{\prime}(t)$ fails to exist at integer multiples of $\pi / 2$, and hence $\eta_{1}$ is nondifferentiable at a countable number of points.

3. Interpolation problems on the conjugate interval $\left[a, \eta_{1}(a)\right]$. It will be shown below that, for almost all points $a$ in the domain of $\eta_{1}$, there is a nontrivial essentially unique solution of the differential equation which has a zero of order $k=$ $\left(k_{0}, \cdots, k_{m}\right)$ at $T=\left\{t_{0}, \cdots, t_{m}\right\}$, where the variables considered satisfy the relations

$$
\begin{aligned}
& p(a)=k_{0}+\cdots+k_{m-1}, \quad q(a)=k_{1}+\cdots+k_{m}, \\
& p(a)+q(a)-n \geq k_{1}+\cdots+k_{m-1}, \quad a=t_{0}<\cdots<t_{m}=\eta_{1}(a) .
\end{aligned}
$$

The question considered here for the extremal integer $p(a)$ appears to be oriented towards maximation of the number of zeros at $a$ of solutions of $L x=0$ having a zero of order $k,|k| \geq n$, at $\left\{a, \eta_{1}(a)\right\}$. If instead one maximizes at $\eta_{1}(a)$, one obtains an "extremal integer" $p^{*}(a)$. All results for $p(a)$ extend to results for $p^{*}(a)$.

The duality that appears here is no accident, and its peculiarity is explained by the following lemma, which follows from the Lagrange identity.

3.1. Lemma. The extremal integer for the adjoint of $L x=0$ is $p^{*}(a)$.

Let us introduce $q^{*}(a)$ just as $q(a)$ was introduced for the extremal integer $p(a)$.

3.2. Remark. One can easily verify that, if $x(t)$ is a solution of $L y=0$ with a zero of order $k=\left(k_{0}, \cdots, k_{m}\right)$ at $T=\left\{a=t_{0}, t_{1}, \cdots, t_{m}=\eta_{1}(a)\right\}$, and

(i) $|k|-k_{m} \geq 1+p(a),|k| \geq n$, or

(ii) $|k|-k_{0} \geq 1+p^{*}(a),|k| \geq n$, then $x(t) \equiv 0$. These facts will be used repeatedly.

The following lemmas are fundamental to the investigation of interpolation problems on the conjugate interval $\left[a, \eta_{1}(a)\right]$; proofs appear in article 6 .

3.3. Lemma. Suppose $p(a)+v>n$, and there is a solution $x$ of (1. 1) with a zero of order exactly $k=(p(a)-u, u, v)$ at $T=\left\{a, b, \eta_{1}(a)\right\}$.

If $T_{n}=\left(t_{n}, b, \eta_{1}\left(t_{n}\right)\right) \rightarrow T$ and $L y=0$ bas a solution $x_{n} \not \equiv 0$ with a zero of order $k$ at $T_{n}$, then there is a solution $y \equiv 0$ of $L y=0$ with a zero of order $(p(a)-x-1, u+1, v-1)$ at $T$. 
Further, $y$ is essentially unique, and if

$$
\lim _{k \rightarrow \infty} \inf _{\eta_{1}\left(t_{k}\right)-\eta_{1}(a)}
$$

is positive, then $y$ 'jas exactly $p(a)+v-1$ zeros on $\left[a, \eta_{1}(a)\right]$.

3.4. Lemma. Suppose $p(a)+v \geq r, u>0$, and $L y=0$ bas a solution $x \equiv 0$ with a zero of order $k=(p(a)-u, u, v)$ at $T=\left\{a, b, \eta_{1}(a)\right\}$.

If $T_{n}=\left\{t_{n}, b, \eta_{1}\left(t_{n}\right)\right\} \rightarrow T, L y=0$ bas a solution $x_{n}$ with a zero of $K$ at $T_{n}$, and $\lim _{\inf _{k \rightarrow \infty}}\left(\eta_{1}\left(t_{k}\right)-\eta_{1}(a)\right) /\left(t_{k}-a\right)>0$, then $L y=0$ bas an essentially unique solution $y \equiv 0$ with a.zero of order $(p(a)-u+1, u-1, v+1)$ at $T$.

Let us call a point $a$ in the domain of $\eta_{1}$ distinguisbed if it satisfies the following condition:

$\{t: p(t)=p(a), q(t)=q(a)\}$ is dense in a neighborhood $U$ of $a$, and $\eta_{1}^{\prime}(a)$ exists and is positive.

Suppose that the distinguished points are not dense in $U$. Then there exists a segment $V \subseteq U$ and a point $b \in V$ such that $p(b)=p(a), q(b)=q(a)$, and $\eta_{1}^{\prime}(t)$ either fails to exist or is zero for all $t \in V$. By the lemma on the differentiability of $\eta_{1}$, we can assume that $\eta_{1} \in C^{2}(V)$. Hence $\eta_{1}^{\prime}(t) \equiv 0$ on $V$. This means that $\eta_{1}$ is constant on $V$. However, Sherman [10] has shown that $\eta_{1}$ is strictly increasing. This proves

3.5. Lemma. The distinguished points are dense in the domain of $\eta_{1}$.

From the preceding lemmas, one can draw the following conclusions; proofs appear in article 6 .

3.6. Theorem. Let $a$ be a distinguished point, and let $b \in\left(a, \eta_{1}(a)\right)$.

If $r$ is any integer satisfying $0 \leq r \leq p(a)+q(a)-n$, then $L y=0$ bas an essentially unique solution $y_{r}$ with a zero of order $(p(a)-r, r, q(a)-r)$ at $\{a, b$, $\left.\eta_{1}(a)\right\}$.

3.7. Theorem. Let $a$ be a distinguished point, and suppose $k=\left(k_{0}, \cdots, k_{m}\right)$, $T=\left\{t_{0}, \cdots, t_{m}\right\}$ with

$$
\begin{aligned}
& p(a)=k_{0}+\cdots+k_{m-1}, \quad q(a)=k_{1}+\cdots+k_{m}, \\
& p(a)+q(a)-n \geq k_{1}+\cdots+k_{m-1}, \quad a=t_{0}<\cdots<t_{m}=\eta_{1}(a) .
\end{aligned}
$$

Then Ly $=0$ bas an essentially unique solution $y(t) \equiv 0$ with a zero of order $k$ at $T$, and $y(t)$ bas exactly $|k|$ zeros on $\left[a, \eta_{1}(a)\right]$. 
In a similar fashion, one can prove the same theorem for $p$ replaced by $q^{*}, q$ replaced by $p^{*}$. However, the distinguished point $a$ must be replaced by its proper analog. One must be careful not to confuse $p^{*}(a)$ and $q^{*}(a)$ with the numbers arising from the adjoint equation. It is true that $p^{*}(a)$ is the extremal integer for $L^{*} y=0$, but this is the most that can be said. Indeed, if $L^{*} y=$ $W(y, 1, \sin t \sin 2 t, \cos t \sin 2 t)$, then $L y=0$ has a solution with a zero of order $(2,2)$ at $\{0, \pi / 2\}$, but $L^{*} y=0$ only has solutions with zeros of order $(2,1)$ and $(1,2)$ at $\{0, \pi / 2\}$. In this case, $p(0)=p^{*}(0)=q(0)=q^{*}(0)=2$, whereas for $L^{*} y=0$ one has $p(0)=p^{*}(0)=2$ and $q(0)=q^{*}(0)=1$.

3.8. Theorem. Except for points a belonging to a nowbere dense set of measure zero, $L y=0$ has an essentially unique solution with a zero of order $k$ at $T$, where $k$ and $T$ satisfy either of the following conditions:

(i) $a=t_{0}<\cdots<t_{m}=\eta_{1}(a), p(a)=|k|-k m, q(a)=|k|-k_{0}, p(a)+q(a)-n \geq$ $|k|-k_{0}-k_{m}$; or

(ii) $a=t_{0}<\ldots<t_{m}=\eta_{1}(a), p^{*}(\dot{a})=|k|-k_{0}, q^{*}(a)=|k|-k_{m}, p^{*}(a)+q^{*}(a)-$ $n \geq|k|-k_{0}-k_{m}$

For $n=3$, Azbelev and Caljuk [1] falsely claimed the existence of solutions with zeros of orders $(2,1)$ and $(1,2)$ at $\left\{a, \eta_{1}(a)\right\}$ implies the existence of a solution with a zero of order $(2,2)$ at $\left\{a, \eta_{1}(a)\right\}$. The following sequence of theorems shows that such boundary value problem behavior is correct almost everywhere.

To illustrate the results further, consider a 6 th order equation with the following property (constant equations serve as examples): for each $a \in(c, d)$ and each $b \in\left(a, \eta_{1}(a)\right)$, there is a solution of $L y=0$ with a zero of order $(1,4,1)$ at $\left\{a, b, \eta_{1}(a)\right\}$. The results say that $L y=0$ has solutions with zeros of orders (1, $4,1),(2,3,2),(3,2,3),(4,1,4),(5,0,5)$ at $\left\{a, b, \eta_{1}(a)\right\}$. Further, $L y=0$ has solutions with a zero of order $k$ at $T$, provided the number of zeros in $\left[a, \eta_{1}(a)\right)$ or $\left(a, \eta_{1}(a)\right]$ does not exceed 5 .

3.9. Theorem. Let $a$ be a distinguished point, $U$ a neighborbood of $a, b \in$ $\left(a, \eta_{1}(a)\right)-U$, and for each $t_{0} \in U$ there is a sequence $\left\{t_{k}\right\}$ converging to $t_{0}\left(t_{k} \neq t_{0}\right)$ with the property that Ly=0 bas a solution with a zero of order $(p(a)-u, u, v)$ at $\left\{t_{k}, b, \eta_{1}\left(t_{k}\right)\right\}$.

If $u>0, p(a)+v \geq n$, then Ly $=0$ has a solution with a zero of order $k$ at $T$, where the variables involved satisfy the relations

$$
\begin{aligned}
& a=t_{0}<\cdots<t_{m}=\eta_{1}(a), \\
& p(a)=|k|-k_{m}, \quad v+u=|k|-k_{0}, \\
& p(a)+u+v-n \geq|k|-k_{0}-k_{m} .
\end{aligned}
$$


3.10. Theorem. There exists a nowbere dense set $A$ of zero measure with the following property: for all $a \notin A$, the existence of a solution of Ly=0 with a zero of order $(p(a)-u, u, v)$ at $\left\{a, b, \eta_{1}(a)\right\}$ for all $b \in\left(a, \eta_{1}(a)\right)$ implies that $L y=0$ bas a solution with a zero of order $k$ at $T$, where the variables involved satisfy relations $(*)$.

4. Applications to equations with constant coefficients. Equations with constant coefficients do not exhibit singular behavior on $\left[a, \eta_{1}(a)\right]$, and the "almost everywhere" results of the preceding sections can be applied at every point.

4.1. Lemma. Suppose $L y=0$ has constant coefficients. Then

(i) If $y(t) \equiv 0$ bas a zero of order $k=\left(k_{0}, \cdots, k_{m}\right)$ at $T=\left\{t_{0}, \cdots, t_{m}\right\}$, then for any $t^{*} \in(-\infty, \infty)$, the solution $y\left(t-t^{*}\right)$ bas a zero of order $k$ at $t^{*}+T \equiv$ $\left\{t^{*}+t_{0}, \cdots, t^{*}+t_{m}\right\}$.

(ii) The domain of $\eta_{1}(t)$ is $(-\infty, \infty)$, unless $L y=0$ is nonoscillatory.

(iii) $\eta_{1}(t)=t+\eta_{1}(0)$.

(iv) $p(t)$ and $q(t)$ are constant functions.

4.2. Theorem. Let $L y=0$ bave constant coefficients. Given arbitrary $a=t_{0}$ $<t_{1}<\cdots<t_{m}=\eta_{1}(a), k=\left(k_{0}, \cdots, k_{m}\right), T=\left\{t_{0}, \cdots, t_{m}\right\}$ with $p+q-n \geq k_{1}+$ $\cdots+k_{m-1}, p=|k|-k_{m}, q=|k|-k_{0}$, there exists an essentially unique solution with a zero of order exactly $k$ at $T$.

A method for the systematic determination of the numbers $p, q, \eta_{1}(0)$ will be sketched below. The author has found the method to be reasonably practical for generating examples and counterexamples in the oscillation theory of higher order equations.

Let $L y=0$ have characteristic polynomial $p(x)=0$ with complex roots $a_{j}$ of multiplicity $n_{j}, j=1, \cdots, k$. Number the fundamental solutions $u_{1}, \cdots, u_{n}$ so that the first $n_{1}$ solutions are $t^{r} \exp (a, t), 0 \leq r \leq n_{1}-1$, the next $n_{2}$ solutions are $t^{r} \exp \left(a_{2} t\right), 0 \leq r \leq n_{2}-1$, etc.

Define $W(t)=W\left(u_{1}, \cdots, u_{n}\right)$ and let $Y_{r}(t)$ be the determinant formed from the first $r$ rows of $W(0)$ and the first $n-r$ rows of $W(t), 1 \leq r \leq n-1$.

To determine $\eta_{1}(t)$ let $b_{r}$ denote the least positive root of $Y_{r}(t)=0$, if such exists, and set $b_{r}=\infty$ otherwise. Then $\eta_{1}(0)=\min \left\{b_{1}, \cdots, b_{n-1}\right\}$, and by the lemma above, $\eta_{1} \stackrel{r}{(t)}=t+\eta_{1}(0)$.

To determine $p$ and $q$, observe that $p=\max \left\{r: b_{r}=\eta_{1}(0)\right\}$; to find $q$, define $X_{r}(s, t)$ to be the $n \times n$ determinant constructed from the first $p$ rows of $w(s)$, the first $n-p-1$ rows of $W(t)$, and the $r$ th row of $W(t), 1 \leq r \leq n-1$. It is easy to verify that $q=\max \left\{v: X_{r}\left(0, \eta_{1}(0)\right)=0\right.$ for $\left.1 \leq r \leq v\right\}$.

Some remarks can be made here which considerably reduce the labor involved. To form $X(0, t)$, write down the first column, which has form

$$
x(a, t)=\left[1, a, \cdots, a^{p-1}, a e^{a t}, \cdots, a^{n-p-1} e^{a t}, a^{r-1} e^{a t}\right]^{T} .
$$


Then the first $\eta_{1}$ columns of $X_{r}(0, t)$ are $(\partial / \partial a)^{k} x(a, t), 0 \leq k \leq \eta_{1}-1$. Similar statements hold for the remaining columns. This procedure works equally well for $Y_{r}(t)$.

5. Applications to $n$th order equations generated from second order equations. An $n$th order equation $L y=0$ is said to be generated from a second order equation $E x=0$ if and only if every solution $x$ of $E x=0$ satisfies $L\left(x^{n-1}\right)=0$. Litera ture on such equations is reasonably plentiful, and the reader is referred to Seda's work ([6], [7], [8]), and the references therein.

The uniqueness of $L y=0$ is a consequence of the following: if $u$ and $v$ are fundamental solutions for $E x=0$, then $\left\{v^{r} u^{n-r-1}\right\}_{r=0}^{n-1}$ is a fundamental set of solutions for $L y=0$. To prove this statement, let $x(t)=u(t)+p v(t)$; then $0=L\left(x^{n-1}\right)$ $=\Sigma_{r=0}^{n-1} C_{r} p^{r}$, where $C_{r}=\left(\begin{array}{c}n-1 \\ r\end{array}\right) L\left(v^{r} u^{n-r-1}\right)$. A polynomial vanishes identically only in case all coefficients vanish, and hence $L\left(v^{r} u^{n-r-1}\right)=0,0 \leq r \leq n-1$. Put $x_{r}(t)=v^{r}(t) u^{n-r-1}(t), 0 \leq r \leq n-1$. The uniqueness follows if $W\left(x_{0}, \cdots, x_{n-1}\right)$ is never zero.

To prove that $W\left(x_{0}, \cdots, x_{n-1}\right)$ is never zero, the identity

$$
\frac{W_{k+1}^{2}}{W_{k}} \frac{d}{d t}\left[\frac{W\left(u_{0}, \cdots, u_{k}, u\right)}{W_{k+1}}\right]=W\left(u_{0}, \ldots, u_{k+1}, u\right)
$$

is used, where $W_{k}=W\left(u_{0}, \cdots, u_{k}\right), 0 \leq k \leq n-1$. The identity is proved by considering both sides to be differential equations in $\mu$. One calculates

$$
W\left(u^{p} v^{m-p}, u^{q} v^{m-q}\right)=(q-p) u^{p+q-1} v^{2 m-p-q-1} W(v, u) .
$$

By induction it can be verified that nonzero constants $A_{q p}, 0 \leq p \leq q$, can be found satisfying

$$
\begin{aligned}
W\left(x_{0}, \ldots, x_{p}\right) & =A_{p p} v^{(p+1)(n-p-1)} W^{p}(v, u), \\
W\left(x_{0}, \ldots, x_{p-1}, x_{q}\right) & =A_{q p}(u / v)^{q-p_{W}}\left(x_{0}, \ldots, x_{p}\right) .
\end{aligned}
$$

In particular, these identities are satisfied for $p=n-1$, and $w\left(x_{0}, \cdots, x_{n-1}\right)$ is a nonzero constant multiple of $W^{n-1}(u, v)$. Since $u$ and $v$ are fundamental for $E x=0, W\left(x_{0}, \cdots, x_{n-1}\right)$ is never zero.

Therefore, $L y=W\left(y, x_{0}, \cdots, x_{n-1}\right) / W\left(x_{0}, \cdots, x_{n-1}\right)$, and this representation is independent of the choice of basis $\{u, v\}$.

5.1. Lemma. Let $L y=0$ be generated from $E x=0$. Then

(a) $L y=0$ and $E x=0$ bave the same conjugate function $\eta_{1}(t)$, and $\eta_{1} \in C^{2}$, $\eta_{1}^{\prime}(t)>0$.

(b) $p(a)=q(a)=n-1$, whenever $\eta_{1}(a)$ exists.

(c) $L=L^{*}$ if and only if $E=E^{*}$. 
5.2. Lemma. Let $u$ be any nontrivial solution of $x^{\prime \prime}+q(t) x=0$, and put $v=$ $u^{\prime}, x=u^{m}, Q_{r}=m(m-1) \cdots(m-r-1) u^{m-r} v^{r}$, and $y^{r}=r(m-r+1) q x^{(r-1)}, r=$ $1,2, y^{r}=r(m-r+1) q\left[x^{(r-1)}+\Sigma_{s=1}^{r-2} y_{s}^{(r-s-2)}\right]$ for $r \geq 3$. Then $L z \equiv y^{(m+1)}+$ $\sum_{r=1}^{m} y_{r}^{(m-r)}=0$ is the equation of order $m+1$ generated from $x^{\prime \prime}+q(t) x=0$.

5.3. Example. The first three equations generated from $x^{\prime \prime}+q(t) x=0$ are given by

$$
\begin{aligned}
& y^{\prime \prime \prime}+4 q y^{\prime}+2 q^{\prime} y=0 \\
& y^{\mathrm{iv}}+10 q y^{\prime \prime}+10 q^{\prime} y^{\prime}+\left(9 q^{2}+3 q^{\prime \prime}\right) y=0 \\
& y^{\nabla}+20 q y^{\prime \prime \prime}+30 q^{\prime} y^{\prime \prime}+\left(18 q+64 q^{2}\right) y^{\prime}+\left(4 q^{\prime \prime}+64 q q^{\prime}\right) y=0
\end{aligned}
$$

By Peano's Theorem (see [4, p. 95]), the fundamental solutions $u_{0}(t, s), \ldots$, $u_{n-1}(t, s)$ defined earlier satisfy the relations

$$
\begin{aligned}
& \partial u_{0}(t, s) / \partial s=p_{0}(s) u_{n-1}(t, s), \\
& \partial u_{k}(t, s) / \partial s=p_{k}(s) u_{n-1}(t, s)-u_{k-1}(t, s), \quad 1 \leq k \leq n-1 .
\end{aligned}
$$

Here, $L y=y^{(n)}+p_{n-1}(t) y^{(n-1)}+\cdots+p_{0}(t) y$. Let $F_{q}(t)$ be the vector of length $q$ with components $u_{q}^{(i)}\left(\eta_{1}(t), t\right), 0 \leq i \leq q-1,1 \leq q \leq n-1$. The above results and a short induction argument show that $F_{q}(t) \equiv 0$ for $1 \leq q \leq n-1$. Hence we the following:

5.4. Theorem. Let $L y=0$ be generated from $E x=0$. Then $u_{k}(t, a)$ bas a zero of order $(k, k)$ at $\left\{a, \eta_{1}(a)\right\}, 0 \leq k \leq n-1$.

5.5. Corollary. The values of $u_{k}(s, t)$ at $s=\eta_{1}(t)$ duplicate according to the relation

$$
u_{n-k+1}^{(p)}\left(\eta_{1}(t), t\right)=\left(\eta_{1}^{\prime}(t)\right)^{k} u_{n-1}^{(p+k)}\left(\eta_{1}(t), t\right)
$$

for $p+k \leq n-1$. Therefore, $u_{n-k-1}(s, t)$ bas an odd or even number of zeros in $\left(t, \eta_{1}(t)\right)$, accordingly as $k$ is even or odd.

If one applies the theorems of the preceding section, then the following results are obtained:

5.6. Theorem. If $L y=0$ is generated from $E x=0, k=\left(k_{0}, \cdots, k_{m}\right), T=$ $\left\{t_{0}, \cdots, t_{m}\right\}, a=t_{0}<t_{1}<\cdots<t_{m}=\eta_{1}(a), n-1=|k|-k_{m}=|k|-k_{0}, k_{1}+\cdots+$ $k_{m-1} \leq n-2$, then there exists an essentially unique solution of $L y=0$ with $a$ zero of order exactly $k$ at $T$.

5.7. Corollary. For any point a, there exists an essentially unique solution with $n$ simple zeros on $\left[a, \eta_{1}(a)\right]$. Further, the interior zeros can be assigned arbitrarily. 
5.8. Theorem. Define $\eta_{1}^{p}(t)=\eta_{1}\left(\eta_{1}^{p-1}(t)\right), \eta_{1}^{0}(t)=t$. If $t_{0}<t_{1}<\cdots<t_{m}=$ $\eta_{1}\left(t_{0}\right), T=\left\{t_{0}, \cdots, t_{m}\right\}, k=\left(k_{0}, \cdots, k_{m}\right)$ and $y(t)$ is an essentially unique solution of $L y=0$ (generated from $E x=0$ ) with a zero of order exactly $k$ at $T$, then $y(t)$ is an essentially unique solution of $L y=0$ with a zero of order exactly $k$ at $\eta_{1}^{p}(T)=\left\{\eta_{1}^{p}\left(t_{0}\right), \cdots, \eta_{1}^{p}\left(t_{m}\right)\right\}$ for $p=0, \pm 1, \pm 2, \cdots$.

5.9. Corollary. Given $\eta_{1}(t)$ and $L y=0$ (generated from $E x=0$ ), and the zeros of $y(t)$ on $\left[0, \eta_{1}(0)\right]$, all other zeros of $y(t)$ on $(-\infty, \infty)$ are known.

5.10. Corollary. Given $\eta_{1}(t)$ with domain $(-\infty, \infty)$ and $L y=0$ (generated from $E x=0)$ of order $n$, all solutions with one zero are oscillatory for $n$ odd, and all solutions are oscillatory for $n$ even.

5.11. Example. The functions $\eta_{1}(t), p(t), q(t)$ do not determine the equation $L y=0$.

Let $c$ be any real number, and put $a=c+i, b=c+3 i$, and define

$$
L_{c} y=(D-a)(D-\bar{a})(D-b)(D-\bar{b}) y
$$

where $D=d / d t$. It is possible, but tedious, to verify that $L_{c} y=0$ is generated from $E_{c} x \equiv x^{\prime \prime}+p_{c} x^{\prime}+q_{c} x=0$ where $p_{c}, q_{c}$ satisfy the relations $3 p_{c}=-2 c$, $11 p_{c}^{2}+10 q_{c}=6 c^{2}+10$. Further, the conjugate functions for all equations are the same, and hence $\eta_{1}(t)=t+\pi$ by consideration of the equation $E_{c} x=0$ for $c=0$. By theorems from this section, $p(a)=q(a)=3$ for all $a$.

5.12. Example. Consider the third order equation

$$
L y \equiv(D-a)(D-b)(D-\bar{b}) y=0
$$

with $a$ real and $\operatorname{Im}(b)>0$. The following assertions hold:

(i) If $\operatorname{Re}(a-b)>0$, then $p(t)=1, q(t)=2$ for all $t$.

(ii) If $\operatorname{Re}(a-b)=0$, then $p(t)=q(t)=2$ for all $t$, and $\eta_{1}(t)=t+2 \pi / \operatorname{Im}(b)$.

(iii) If $\operatorname{Re}(a-b)<0$, then $p(t)=2, q(t)=1$ for all $t$.

A consequence of this example is that there exists infinitely many third order equations with the same functions $p(t), q(t), \eta_{1}(t)$.

6. Proofs of the theorems.

Proof of Lemma 2.4. Let $b=\eta_{1}(a)$. Define $G_{k}(t)$ and $H_{k}(t)$ as follows: $G_{k}(t)$ is the matrix formed by the first $k$ rows of $W\left(u_{0}(t, a), \cdots, u_{n-1}(t, a)\right)$, and $H_{k}(t)$ is the $k$ th row, $1 \leq k \leq n$. Define $F(t, s)$ to be the determinant of the $n \times n$ matrix formed from the matrices $G_{p(a)}(t), G_{n-p(a)-1}(s), H_{q(a)}(s)$. A submatrix is omitted if its subscript is zero.

It will be shown that the implicit function theorem is applicable to the function $F(t, s)$. Since there is a nontrivial solution $x$ of the equation with a zero of order $(p(a), q(a))$ at $\{a, b\}$, we have $F(a, b)=0$. If $F_{s}(a, b)=0$, then there is a 
nontrivial solution $y$ with a zero of $\operatorname{order}(p(a), n-p(a)-1)$ at $\{a, b\}$ with $D^{q(a)} y(b)=0$. Let

$$
z(t)=D^{p(a)} x(a) y(t)-D^{p(a)} y(a) x(t) .
$$

Since $x$ and $y$ are linearly independent, $z \not \equiv 0$. Further, $z$ is a solution with a zero of order $(p(a)+1, n-p(a)-1)$ at $\{a, b\}$. This contradicts the definition of $p(a)$, and one concludes that $F_{s}(a, b) \neq 0$.

The implicit function theorem says that there is a neighborhood $L$ of $t=a$ and a function $g \in C(L)$ with $g(a)=b$ and $F(t, g(t))=0$ for $t \in L$. By uniqueness and the continuity of $\eta_{1}(t)$, the relation $F\left(t, \eta_{1}(t)\right)=0$ implies that $g=\eta_{1}$ in a small neighborhood of $t=a$. The function $F$ is of class $C^{2}$, so $g$ is of class $C^{2}$. Hence, $\eta_{1} \in C^{2}(U)$ for some neighborhood $U$ of $t=a . \square$

Proof of Corollary 2.6. The theorem implies that we only have to show the set $V=\left\{t: \eta_{1}(t), \eta_{1}^{\prime}(t)\right.$ exist, $\left.\eta_{1}^{\prime}(t)=0\right\}$ is nowhere dense and has measure zero. Actually, $V$ is a finite union of discrete sets, as will be shown below.

Let $J_{u v}$ be the set of all points $t_{0} \in V$ such that $L y=0$ has an essentially unique solution with a zero of order exactly $(u, v)$ at $\left\{t_{0}, \eta_{1}\left(t_{0}\right)\right\}$, but no nontrivial solution of $L y=0$ has $n$ zeros on $\left[t_{0}, \eta_{1}\left(t_{0}\right)\right]$ and $v+1$ zeros on $\left(t_{0}, \eta_{1}\left(t_{0}\right)\right]$, i.e., $v$ is the maximum number of zeros on $\left(t_{0}, \eta_{1}\left(t_{0}\right)\right]$ for any nontrivial solution with $n$ zeros on $\left[t_{0}, \eta_{1}\left(t_{0}\right)\right]$.

The set $V$ is the union of the sets $J_{u v}$; this can be verified through use of the adjoint equation. It will be shown that each $J_{u v}$ is discrete. Let $F(t, s)$ be the determinant whose first $n-v$ rows are the first $n-v-1$ rows, and row $u$, of $W\left(u_{0}\left(t, t_{0}\right), \cdots, u_{n-1}\left(t, t_{0}\right)\right)$, and whose last $v$ rows are the first $v$ rows of $W\left(u_{0}\left(s, t_{0}\right), \cdots, u_{n-1}\left(s, t_{0}\right)\right)$. If $t_{0} \in J_{u v}$ and every neighborhood of $t_{0}$ meets $J_{u v}$, then there exist solutions $x_{n}(t) \not 0$ and points $t_{n} \neq t_{0}$ such that $t_{n} \rightarrow t_{0}$ as $n \rightarrow \infty$, and $x_{n}(t)$ has a zero of order $(u, v)$ at $\left\{t_{n}, \eta_{1}\left(t_{n}{ }_{n}\right)\right\}, n \geq 1$. Therefore, $F\left(t_{n}, \eta_{1}\left(t_{n}\right)\right)$ $=0, n \geq 0$, and it follows that

$$
\eta_{1}^{\prime}\left(t_{0}\right) F_{s}\left(t_{0}, \eta_{1}\left(t_{0}\right)\right)+F_{t}\left(t_{0}, \eta_{1}\left(t_{0}\right)\right)=0
$$

Since $\eta_{1}^{\prime}\left(t_{0}\right)=0$, one has $F_{t}\left(t_{0}, \eta_{1}\left(t_{0}\right)\right)=0$. Therefore, there exists a solution $x(t) \not 0$ of $L x=0$ with a zero of order $(n-v-1, v)$ at $\left\{t_{0}, \eta_{1}\left(t_{0}\right)\right\}$ with $x^{(u)}\left(t_{0}\right)$ $=0$. The solution $y(t) \not 0$ with a zero of order exactly $(u, v)$ at $\left\{t_{0}, \eta_{1}\left(t_{0}\right)\right\}$ is essentially unique, so $x$ and $y$ are linearly independent. Further, $t_{0} \in J_{u v}$ implies $x^{(v)}\left(\eta_{1}\left(t_{0}\right)\right) \neq 0$ and $y^{(v)}\left(\eta_{1}\left(t_{0}\right)\right) \neq 0$. Therefore, $z(t)=x^{(v)}\left(\eta_{1}\left(t_{0}\right)\right) y(t)-$ $y^{(v)}\left(\eta_{1}\left(t_{0}\right)\right) x(t) \equiv 0$. However, $z(t)$ has a zero of order $(n-v-1, v+1)$ at $\left\{t_{0}\right.$, $\left.\eta_{1}\left(t_{0}\right)\right\}$, which contradicts $t_{0} \in J_{u v}$. One concludes that $t_{0}$ has a deleted neighborhood which misses $J_{u v}$. Therefore, $J_{u v}$ is discrete, as claimed. Consequently $V$ is a finite union of discrete sets, and hence $V$ is nowhere dense and of zero measure. $\square$ 
Proof of Lemma 3.3. Let $W(t)=W\left(u_{0}(t, a), \cdots, u_{n-1}(t, a)\right)$, define $G_{k}(t)$ to be the $k \times n$ matrix formed from the first $k$ rows of $W(t)$, and put $H_{k}(t)$ equal to the $k$ th row of $W(t), 1 \leq k \leq n$. Put $p=p(a), c=\eta_{1}(a)$, and let

$$
F_{k}(t)=\operatorname{det}\left[\begin{array}{l}
G_{p-u}(t) \\
G_{u}(b) \\
G_{n-p-1}(s) \\
H_{k}(s)
\end{array}\right] \quad(1 \leq k \leq v) .
$$

A matrix is to be omitted in this formula if its subscript is zero. The relation $F_{v}(a, c)=0$ follows from the hypothesis that $x(t)$ has a zero of order $k$ at $T$. In order to prove that $\partial F_{v}(a, c) / \partial s \neq 0$, let us assume the contrary; then there exists a solution $z \equiv 0$ of $L z=0$ with a zero of order $(p-u, u, n-p-1)$ at $T$ with $z^{(v)}(c)=0$. A linear combination of $x$ and $z$ has a zero of order $(p-u, u+1, n-$ $p-1)$ at $T$. However, this solution has $n$ zeros in $\left[a, \eta_{1}(a)\right]$ and $p+1$ zeros in $\left[a, \eta_{1}(a)\right)$. By previous remarks, this solution vanishes identically. Accordingly, $\partial F_{v}(a, c) / \partial s \neq 0$.

The implicit function theorem implies there is a neighborhood $U$ of $a$ and a real-valued function $g \in C^{2}(U)$ such that $g(a)=c$ and $F_{v}(t, g(t))=0$ for all $t \in U$. The weak uniqueness assertion together with $F_{v}\left(t_{k}, \eta_{1}\left(t_{k}\right)\right)=0$ implies $g\left(t_{k}\right)=$ $\eta_{1}\left(t_{k}\right)$ for all large $K$.

Define $E_{j}(t)=F_{j}(t, g(t)), t \in U, 1 \leq j \leq v$. The function $E_{j}(t)$ vanishes for $t=t_{k}, k$ large, and therefore $E_{j}^{\prime}(a)=0,1 \leq j \leq v$. The formula for $F_{j}(t, g(t))$ is differentiated at $t=a$, and one obtains for $j+1 \leq v$

$$
0=E_{j}^{\prime}(a)=\operatorname{det}\left[\begin{array}{l}
G_{p-u-1}(a) \\
H_{p-u+1}(a) \\
G_{u}^{(b)} \\
G_{n-p-1}(c) \\
H_{j}(c)
\end{array}\right]+g^{\prime}(a) \operatorname{det}\left[\begin{array}{l}
G_{p-u^{(a)}} \\
G_{n}^{(b)} \\
G_{n-p-2^{(c)}} \\
H_{n-p}(c) \\
H_{j}(c)
\end{array}\right]+g^{\prime}(a) \operatorname{det}\left[\begin{array}{l}
G_{p-u}(a) \\
G_{u}^{(b)} \\
G_{n-p-1}(c) \\
H_{j+1}(c)
\end{array}\right]
$$

$$
=\operatorname{det}\left[\begin{array}{l}
G_{p-u-1}(a) \\
H_{p-u+1}^{(a)} \\
G_{u}^{(b)} \\
G_{n-p-1}^{(c)} \\
H_{j}(c)
\end{array}\right]
$$

Hence, for $1 \leq j \leq v-1$, there exist solutions $x_{j}(t) \not \equiv 0$ with a zero of order $(p-u-1, u, n-p-1)$ at $T$, and $x_{j}^{(p-u)}(a)=x_{j}^{(j-1)}(c)=0$. 
It will be shown that $x_{1}(t), \ldots, x_{v-1}(t)$ are constant multiples of a single solution $y_{0}(t) \not 0$. It suffices to prove that $x_{j}=a_{j} x_{j+1}$ for some $\alpha_{j} \neq 0,1 \leq j \leq$ $v-1$. First, one observes that $x_{j}^{(p-u-1)}(a) \neq 0$, for otherwise there would be a nontrivial solution with a zero of order $(p-u+1, u, n-p-1)$ at $T$, an impossibility by (3.2). Therefore, one can put $\alpha_{j}=x_{j}^{(p-u-1)}(a) / x_{j}^{(p-n-1)}(a)$, and by (3.2) one concludes that $x_{j}(t)-\alpha_{j} x_{j+1}(t) \equiv 0$. Thus, there exists a solution $y_{0} \not \equiv$ with a zero of order $(p-u-1, u, v-1)$ at $T$, and $y_{0}^{(p-u)}(a)=0$.

If $y_{0}^{(u)}(b)=0$, then we may put $y(t)=y_{0}(t)$, and $y(t)$ is the desired solution. Otherwise, $y_{0}^{(u)}(b) \neq 0$. Further, $x^{(u)}(b) \neq 0$, for otherwise $x(t)$ would have a zero of order $(p-u, u+1, v)$ at $T$, an impossibility by (3.2). In this case one can take $y(t)=y_{0}(t)+\alpha x(t)$, where $a=-y_{0}^{(u)}(b) / x^{(u)}(b)$.

The solution $y(t)$ is essentially unique, for if $\bar{y}(t)$ is another, then $\bar{y}^{(u+1)}(b) y(t)-y^{(u+1)}(b) \bar{y}(t)$ has a zero of order $(p-u-1, u+2, v-1)$ at $T$, and hence is identically zero by (3.2).

Finally, let us assume that $\lim \inf _{k \rightarrow \infty}\left(\eta_{1}\left(t_{k}\right)-\eta_{1}(a)\right) /\left(t_{k}-a\right)>0$, yet $y(t)$ has at least $(p+v-1)+1$ zeros in $\left[a, \eta_{1}(a)\right]$. Since $g\left(t_{k}\right)=\eta_{1}\left(t_{k}\right)$ for all large $k$, we have $g^{\prime}(a)>0$. By (3.2), $y(t)$ can have no other zeros in $\left[a, \eta_{1}(a)\right)$, and therefore $y(t)$ has $v$ zeros at $c$.

Consider the three determinants which appear in (6.1) for $j=v$. A linear combination $z(t)$ of $x(t)$ and $y(t)$ has a zero of order $(p-u-1, u, v)$ at $T$, and $z^{(p-u)}(a)=0$; hence the first determinant vanishes. Since $x(t)$ has a zero of order $(p-u, u, v)$ at $T$, and $p+v>n$, the second determinant vanishes. Therefore, the last determinant must vanish.

One concludes that there exists a solution of $L y=0$ with a zero of order ( $p-$ $u, u, n-p-1)$ at $T$, and this solution is not a multiple of $x(t)$. An elementary linear combination argument and (3.2) show this to be impossible. Therefore, $y(t)$ has exactly $p+v-1$ zeros on the conjugate interval.

Proof of Lemma 3.4. Let $y_{k}(t)$ be a nontrivial solution of $L y=0$ with a zero of order $(p-u+1, u-1, n-p-1)$ at $T_{k}, k \geq 1$. Renumber the sequence, if necessary, so that $x_{k}(t)$ and $y_{k}(t)$ have exactly $p$ zeros on $\left[t_{k}, \eta_{1}\left(t_{k}\right)\right), k \geq 1$; this can be done by the convergence lemma and Remark (3.2). If the exact order $v_{0}$ of the zero of $x_{k}(t)$ at $\eta_{1}\left(t_{k}\right)$ is less than $v$, then Sherman's Lemma 2.1 shows that $L y=0$ has a nontrivial solution with a zero of order $\left(p-u, u-1,2, v_{0}\right)$ at $\left\{t_{k}, b, c, \eta_{1}\left(t_{k}\right)\right\}$, where $c \in\left(b, \eta_{1}\left(t_{k}\right)\right)$. By (3.2), such a solution cannot exist, and therefore $v_{0} \geq v$. A linear combination of $x_{k}(t)$ and $y_{k}(t)$ has a zero of order $(p-u, u-1, v+1)$ at $T_{k}$, and by the convergence lemma there exists a solution $w(t) \equiv 0$ with a zero of order $(p-u, u-1, v+1)$ at $T$. Further, the convergence lemma applied to $\left\{y_{k}\right\}$ shows there exists a solution $z(t) \equiv 0$ with a zero of order $(p-u+1, u-1, v)$ at $T$. 
Let $r$ be the exact order of the zero of $z(t)$ at $c=\eta_{1}(a)$. It will be shown that $r \geq v+1$.

Suppose not, and let $G_{k}, H_{k}$ be defined as in the previous lemma. Define $F(t, s)$ as the determinant of the matrix formed from $G_{p-u+1}(t), G_{n-1}(b)$, $G_{n-p-1}(s) H_{r}(s)$, as in the previous lemma. A matrix is omitted if its subscript is zero. It is checked that $F \in C^{2}, F(a, c)=0, F_{s}(a, c) \neq 0$. The implicit function theorem implies the existence of a neighborhood $U$ of $a$ and a function $f \epsilon$ $C^{2}(U)$ such that $f(a)=c=\eta_{1}(a)$ and $F(t, f(t))=0$ for all $t \in U$.

Differentiation of $F(t, f(t))=0$ gives $F_{t}(a, c)+f^{\prime}(a) F_{s}(a, c)=0$. The first term of this expression is the determinant of the matrix formed from $G_{p-u}(a)$, $H_{p-u+2}(a), G_{u-1}(b), G_{n-p-1}(c), H_{r}(c)$. The determinant $F_{s}(a, c)$ is the sum of two determinants: the first is formed from $G_{p-u+1}(a), G_{u-1}(b), G_{n-p-2}(c), H_{n-p}(c)$, $H_{r}(c)$, and the second is formed from $G_{p-n+1}(a), G_{u-1}(b), G_{n-p-1}(c), H_{r+1}(c)$. By examination of the solutions $w(t)$ and $z(t)$, and linear combinations of these two solutions, one can verify that the first two determinants just described are zero.

It will be shown that the last determinant vanishes; this will follow if $f^{\prime}(a) \neq 0$. By the weak uniqueness assertion of the Implicit Function Theorem, we have $f\left(t_{k}\right)=\eta_{1}\left(t_{k}\right)$ for all large $K$, because $F\left(t_{k}, \eta_{1}\left(t_{k}\right)\right)=0$, and $t_{k} \rightarrow a$ as $k \rightarrow \infty$. Therefore,

$$
f^{\prime}(a)=\lim _{k \rightarrow \infty} \frac{\eta_{1}\left(t_{k}\right)-\eta_{1}(a)}{t_{k}-a}>0 .
$$

The vanishing of the determinant in question implies the existence of a solution $z_{0}(t) \equiv 0$ with a zero of order $(p-u+1, u-1, n-p-1)$ at $T$ with $z_{0}^{(r)}(c)$ $=0$. Since $r=v, z(t)$ and $z_{0}(t)$ are linearly independent. A linear combination of $z$ and $z_{0}$ has a zero of order $(p-u+1, u, n-p-1)$ at $T$, which contradicts (3.2).

Therefore, $r \geq v+1$, and $z(t)$ has a zero of order $(p-u+1, u-1, v+1)$ at $T$. In view of (3.2), $z(t)$ is essentially unique.

Proof of Theorem 3.6. For $r=0$, the theorem follows from Sherman's result [10]. Let $r \geq 1$ be given and suppose the theorem is true for $r-1$.

There exists a sequence $\left\{t_{k}\right\}_{k=1}^{\infty}, t_{k} \neq a$, with $t_{k} \rightarrow a$ as $k \rightarrow \infty, t_{k}<b<$ $\eta_{1}\left(t_{k}\right)$ for each $k \geq 1$, and $p\left(t_{k}\right)=p(a)$ for all $k$. Further, we may assume that each $t_{k}$ is distinguished.

The induction hypothesis implies that there exists a solution $x_{k}(t) \not \equiv$ with a zero of order $(p-r+1, r-1, q-r+1)$ at $T_{k}=\left\{t_{k}, b, \eta_{1}\left(t_{k}\right)\right\}, k \geq 1$, and a solution with a zero of order exactly $(p-r+1, r-1, q-r+1)$ at $T=\{a, b$, $\left.\eta_{1}(a)\right\}$. Lemma 3.3 shows that there exists a solution $y(t) \not 0$ with a zero of order $(p-r, r, q-r)$ at $T$. Since $a$ is a distinguished point, $y(t)$ has exactly $p+q-r$ 
zeros, and we take $y_{r} \equiv y$ to complete the induction. $\square$

Proof of Theorem 3.7. Let $v_{0}=k_{1}+\cdots+k_{m-1}$. Then $k_{0}=p-v_{0}, k_{m}=q-$ $v_{0}$, and $0 \leq v_{0} \leq p+q-n$. By the previous theorem, there exist solution $y_{r}, k_{1} \leq$ $r \leq v_{0}$, such that $y_{r}$ has a zero of order $(p-r, r, q-r)$ at $\left\{t_{0}, t_{1}, t_{m}\right\}$. Constants $c_{r}, k_{1} \leq r \leq v_{0}$, shall be determined such that

$$
x(t)=\sum_{k_{1} \leq r \leq v_{0}} c_{r} y_{r}(t)
$$

is a nontrivial solution with a zero of order $k$ at $T$.

The function $x(t)$ already has a zero of order $\left(k_{0}, k_{1}, k_{m}\right)$ at $\left\{t_{0}, t_{1}, t_{m}\right\}$, so it suffices to determine conditions on the constants to insure that $x(t)$ has a zero of order $\left(k_{2}, \cdots, k_{m-1}\right)$ at $\left\{t_{2}, \cdots, t_{m-1}\right\}$. If one puts $x(t)$ into the boundary conditions of this problem, then a system of $k_{2}+\cdots+k_{m-1}=v_{0}-k_{1}$ equations in $v_{0}-k_{1}+1$ unknowns results. This system of equations always has a nontrivial solution. Hence, nontrivial constants $c_{r}$ can be found, and $x(t) \not \equiv$ is the desired solution.

It remains to prove that $x(t)$ is essentially unique, and $x(t)$ has exactly $|k|$ zeros on the conjugate interval. To this end, suppose $x_{0}(t)$ is another solution. Since both $x(t)$ and $x_{0}(t)$ have $p$ zeros on $\left[a, \eta_{1}(a)\right)$, and $n$ zeros on the closed interval, (3.2) implies that $x_{0}$ and $x$ are linearly dependent. Thus, $x(t)$ is essentially unique. If $x(t)$ has $|k|+1$ zeros on the closed interval, then the additional zero occurs at $\eta_{1}(a)$ by (3.2). It follows immediately that $x(t)$ is a linear combination of the $y_{r}, k_{1} \leq r \leq v_{0}$. But these solutions all have a zero of order $p-v_{0}+1$ at $a$, and hence $x(t)$ has $p+1$ zeros on $\left[a, \eta_{1}(a)\right)$, contradicting (3.2). Therefore, $x(t)$ has exactly $|k|$ zeros on $\left[a, \eta_{1}(a)\right]$.

Proof of Theorem 3.8. Let $A_{u v}$ denote the set of all points $t$ such that $p(t)=$ $u, q(t)=v$, and

$$
\liminf _{s \rightarrow t} \frac{\eta_{1}(s)-\eta_{1}(t)}{s-t}>0 .
$$

Let $B_{0}=A_{u v}$, and inductively define $B_{k+1}$ to be the set of all $t \in B_{k}$ such that every deleted neighborhood of $t$ meets $B_{k}, k=0,1, \cdots, d, d=u+v-n-1$.

Then

$$
A_{u v}=E_{0} \cup E_{1} \cup \cdots \cup E_{d}
$$

where $E_{d}=B_{d}, E_{k}=B_{k}-B_{k+1}$ for $k<d$.

It will be shown by induction that for any $t \in B_{k}, k=1, \ldots, d$, there is an essentially unique solution with a zero of order exactly $(u-k, k, v-k)$ at $\{t$, $\left.b, \eta_{1}(t)\right\}$, for all $b \in\left(t, \eta_{1}(t)\right)$. For $k=1$, this follows directly from Lemma 3.3. Assume the result for $k$; then for all $t \in B_{k}$ there is an essentially unique solution with a zero of order $(u-k, k, v-k)$ at $\left\{t, b, \eta_{1}(t)\right\}$, for all $b \in\left(t, \eta_{1}(t)\right)$. 
Given any $t_{0} \in B_{k+1}, t_{0}$ is the sequential limit of points $t \in B_{k}$ with $t \neq t_{0}$. Since $t_{0}=B_{0}$, the hypothes is of Lemma 3.3 is satisfied, and therefore there exists an essentially unique solution with a zero of order exactly $(u-k-1, k+1, v-$ $k-1)$ at $\left\{t_{0}, b, \eta_{1}\left(t_{0}\right)\right\}$, for all $b \in\left(t_{0}, \eta_{1}\left(t_{0}\right)\right)$. This completes the induction, and shows that for all $t_{0} \in E_{d}$, there is an essentially unique solution with a zero of order exactly $(u-k, k, v-k)$ at $\left\{t_{0}, b, \eta_{1}\left(t_{0}\right)\right\}$ for all $b \in\left(t_{0}, \eta_{1}\left(t_{0}\right)\right)$ and all $k$ with $0 \leq k \leq u+v-n-1$. The proof of Theorem 3.7 shows that, for all $t_{0} \in E_{d}$, there is an essentially unique solution with a zero of order exactly $k$ at $T$ ( $k$ and $T$ as in the statement of the theorem).

Let $H_{u v}$ denote the union of the sets $E_{k}, 0 \leq k \leq d-1, K_{u v}=E_{d}$, and let $M_{u v}$ be the points $t$ such that $p(t)=u, q(t)=v$, and

$$
\underset{s \rightarrow t}{\lim \inf } \frac{\eta_{1}(s)-\eta_{1}(t)}{s-t}=0 .
$$

Define $H, K, M$ as the union of the sets $H_{u v}, K_{u v}, M_{u v}$, respectively. Then $H \cup$ $K \cup M$ is the domain of $\eta_{1}(t)$.

By Corollary 2.6, $M$ is nowhere dense and has zero measure.

It will be shown that $H$ is nowhere dense and has zero measure. Actually, $H$ is a finite union of discrete sets, and this fact will be proven to establish the result. Consider again the sets $E_{0}, E_{1}, \cdots, E_{d-1}$, whose union is $H_{u v}$. If $t \in E_{0}$ and every neighborhood of $t$ meets $E_{0}$, then every neighborhood of $t$ meets $B_{0}$, and hence $t \in B_{1}$. But $E_{0}=B_{0}-B_{1}$, so this is impossible. Accordingly, each point of $E_{0}$ has deleted neighborhood which misses $E_{0}$. Thus $E_{0}$ is discrete. In the same way, one proves that $E_{1}, \cdots, E_{d-1}$ are discrete. Thus $H_{u v}$, and also $H$, is a finite union of discrete sets.

For all $t_{0} \in k$, there is an essentially unique solution of $L y=0$ with a zero of order exactly $k$ at $T$. The complement of $K$ in the domain of $\eta_{1}(t)$ is $H \cup M$, and this is a nowhere dense set of zero measure.

Proof of Theorem 3.9. This result follows from Theorem 3.7, if one can show that the inequality $q(a) \geq u+v$ is a consequence of the given hypotheses.

If Lemma 3.4 is applied to each distinguished point $t_{0} \in U$ with $p\left(t_{0}\right)=p(a)$, then it follows that $L y=0$ has a solution with a zero of order $(p-u+1, u-1$, $v+1)$ at $\left\{t_{0}, b, \eta_{1}\left(t_{0}\right)\right\}$. The points $t_{0}$ with the described property are dense in a neighborhood of $a$, and a standard induction with Lemma 3.4 shows that $L y=0$ has a nontrivial solution with a zero of order $(p, v+u)$ at $\left\{a, \eta_{1}(a)\right\}$. Therefore, $q(a) \geq u+v$, and the result follows from Theorem 3.7.

Proof of Theorem 3.10. The set $K$ of all points $a$ with the property described in the hypothesis of the preceding theorem can be taken to be the complement of $A$, and it only remains to prove that $A$ is nowhere dense and has zero measure. These arguments are tedious, but straightforward, and parallel the proof of Theorem 3.8. 
Proof of Lemma 5.1. Suppose $a^{*}$ is conjugate to $a$ for the equation $E x=0$. It will be shown that $\eta_{1}(a)$ exists for $L y=0$, and equals $a^{*}$. Any solution with $p$ zeros at $a$ has the form

$$
y(t)=\sum_{r=0}^{n-p-1} C_{r} u^{n-r-1}(t) v^{r}(t)
$$

where $u$ and $v$ are solutions of Ex with $u(a)=0$ and $W(v, u) \neq 0$. Since $y_{0}(t)=$ $u^{n-1}(t)$ has $n$ zeros on $\left[a, a^{*}\right], \eta_{1}(a)$ exists, $\eta_{1}(a) \leq a^{*}$. Let $p=p(a)$, and suppose $y(t)$ has a zero of order $(p(a), q(a))$ at $\left\{a, \eta_{1}(a)\right\}$. Therefore, since $q=q(a)$ satisfies $q \geq n-p$, differentiation of $y(t)$ at $\eta_{1}(a)<a^{*}$ gives

$$
W\left(u^{n-p-1}, v u^{n-p-2}, \cdots, v^{n-p-1}\right)=0
$$

at $\eta_{1}(a)$, which has been shown to be impossible when $W(v, u) \neq 0$. Hence $\eta_{1}(a)=a^{*}$. The preceding calculation is valid at $a^{*}$ if $p<n-1$, so $p(a)=n-1$. Then $y(t)=c_{0} u^{n-1}(t)$, and $q(a)=n-1$.

To prove $\eta_{1} \in C^{2}$, and $\eta_{1}^{\prime}(t)>0$, it is convenient to consider the relation $u_{1}\left(t, \eta_{1}(t)\right) \equiv 0$, valid for any second order equation. The theorems of differentiability of $\eta_{1}(t)$ imply $\eta_{1} \in C^{2}$, and the above relation gives $-\eta_{1}^{\prime}(t) u_{0}\left(t, \eta_{1}(t)\right)+$ $u_{1}^{\prime}\left(t, \eta_{1}(t)\right)=0$. Since $u_{1}^{\prime}\left(t, \eta_{1}(t)\right) \neq 0, \eta_{1}^{\prime}(t)>0$.

The statement about formal adjoints is a consequence of the relation

$$
\frac{W\left(x_{0}, \cdots, x_{n-2}\right)}{W\left(x_{0}, \cdots, x_{n-1}\right)}=\frac{c v^{n-1}}{W(v, u)}
$$

where $c \neq 0$ is a constant and $x_{r}=u^{r} v^{n-r-1}$, in conjuction with Liouville's formula (see Hartman [4]).

Proof of Lemma 5.2. This is easily established by induction; uniqueness shows that the recursions do not depend on the choice of $n . \square$

Proof of Theorem 5.4. Since $u_{n-1}(t, a)=c u^{n-1}(t)$ where $u(t)$ is a nontrivial solution of $E x=0$ with $n(a)=0$, the theorem is obviously true for $k=n-1$. The formula $u_{n-1}\left(t, \eta_{1}(t)\right)=0$, together with $\eta_{1}^{\prime}(t)>0$ and simple induction, establishes the result. $\square$

Proof of Corollary 5.5. This is a consequence of the proof of theorem.

Proof of Theorem 5.8. There is nothing to prove for $p=0$. Write $y(t)=$ $\sum_{r=0}^{n-1-k_{1}} c_{r} u_{n-r-1}\left(t, t_{1}\right)$. Then, by Theorem 5.4, $y(t)$ has a zero of order $k_{1}$ at $\eta_{1}\left(t_{1}\right)$. Similarly, $y(t)$ has a zero of order $k_{2}$ at $\eta_{1}\left(t_{2}\right)$. The induction is left tc the reader. $\square$

\section{BIBLIOGRAPHY}

1. N. V. Azbelev and Z. B. Caljuk, On the question of the distribution of the zeros of a third-order linear differential equation, Mat. Sb. 51 (93) (1960), 475-486; English transl., Amer. Math. Soc. Transl. (2) 42 (1964), 233-245. MR 22 \#12266. 
2. J. H. Barrett, Oscillation theory of ordinary linear differential equations, Advances in Math. 3 (1969), 415-509. MR 41 \#2113.

3. J. Dieudonné, Foundations of modern analysis, Pure and Appl. Math., vol. 10, Academic Press, New York, 1960. MR 22 \#11074.

4. P. Hartman, Ordinary differential equations, Wiley, New York, 1969.

5. W. Leighton and Z. Nehari, On the oscillation of solutions of self-adjoint linear differential equations of the fourth order, Trans. Amer. Math. Soc. 89 (1958), 325-377. MR $21 \# 1429$.

6. V. Šeda, Über die Transformation der linearen Differentialgleichungen n-ter Ordnung. I, Časopis P̌est. Mat. 90 (1965), 385-412. MR 33 \#4356.

7. - Über die Transformation der linearen Differentialgleichungen n-ter Ordung. II, Č̀asopis P̌est. Mat 92 (1967), 418-435. MR 37 \#5454.

8. - On a class of linear differential equations of order $n, n \geq 3$, Časopis Р̌est. Mat. 92 (1967), 247-261. MR 36 \#5442.

9. T. L. Sherman, Properties of solutions of nth order linear differential equations, Pacific J. Math. 15 (1965), 1045-1060. MR 32 \#2654.

10. - Conjugate points and simple zeros for ordinary linear differential equations, Trans. Amer. Math. Soc. 146 (1969), 397-411. MR 41 \#572.

DEPARTMENT OF MATHEMATICS, UNIVERSITY OF UTAH, SALT LAKE CITY, UTAH 84112 